\title{
Precession-Nutation Estimates from Optical Astrometry 1899.7-1992.0 and Comparison with VLBI Results
}

\author{
Jan Vondrák and Cyril Ron \\ Astronomical Institute, Academy of Sciences of the Czech Republic, \\ Bočni II, 14131 Prague 4, Czech Republic
}

\begin{abstract}
The most recent solution of Earth orientation parameters from the observations made by optical astrometry in 1899.7-1992.0 at 33 observatories is used to estimate the corrections of the present IAU model of precession-nutation. Since the resolution of the solution is about five days, only the terms with periods of 14 days and longer are considered. The results are compared with VLBI-based corrections of the standard precession-nutation model, and the differences are discussed.
\end{abstract}

\section{Introduction}

Earth Orientation Parameters (EOP - polar motion $x, y$, Universal Time UT1TAI and celestial pole offsets $\Delta \varepsilon, \Delta \psi$ ) have been recently derived from optical astrometry observations made since the beginning of the century at 33 observatories. The Hipparcos Catalog (Kovalevsky et al., 1997) was used to recalculate all the observations; they were thus brought into the recently adopted International Celestial Reference System ICRS (Feissel \& Mignard, 1998). The complete description of the algorithms applied in our preceding solution are described by Vondrák et al. (1998a). Small changes introduced later to produce the most recent solution are given in Vondrák et al. (2000). The changes between these two solutions consist in including data from two more observatories (Mount Stromlo and Józefoslaw), adding more data from Kharkov and Blagoveschtchensk, discarding the data from Ukiah after 1960, and more Hipparcos proper motions corrected than before. These solutions are here denoted as OA97 and OA99.

\section{The solution}

The details of the solution OA99 being described in detail elsewhere, only a short description is given here. The solution, covering the interval 1899.7-1992.0, is based on approximately 4.5 million individual values of three types of observables (instantaneous latitude $\varphi$, difference of Universal Time from the uniform atomic time scale (UT0-TAI) and the difference between observed and calculated zenith distance of the star $\delta h$ ), according to the type of instrument used. The observations were made with 50 different instruments at 33 observatories. In case two or more similar instruments were used at the same observatory, their results were merged into a single series, with the steps due to different locations of the instruments removed, and treated further as a single instrument. 

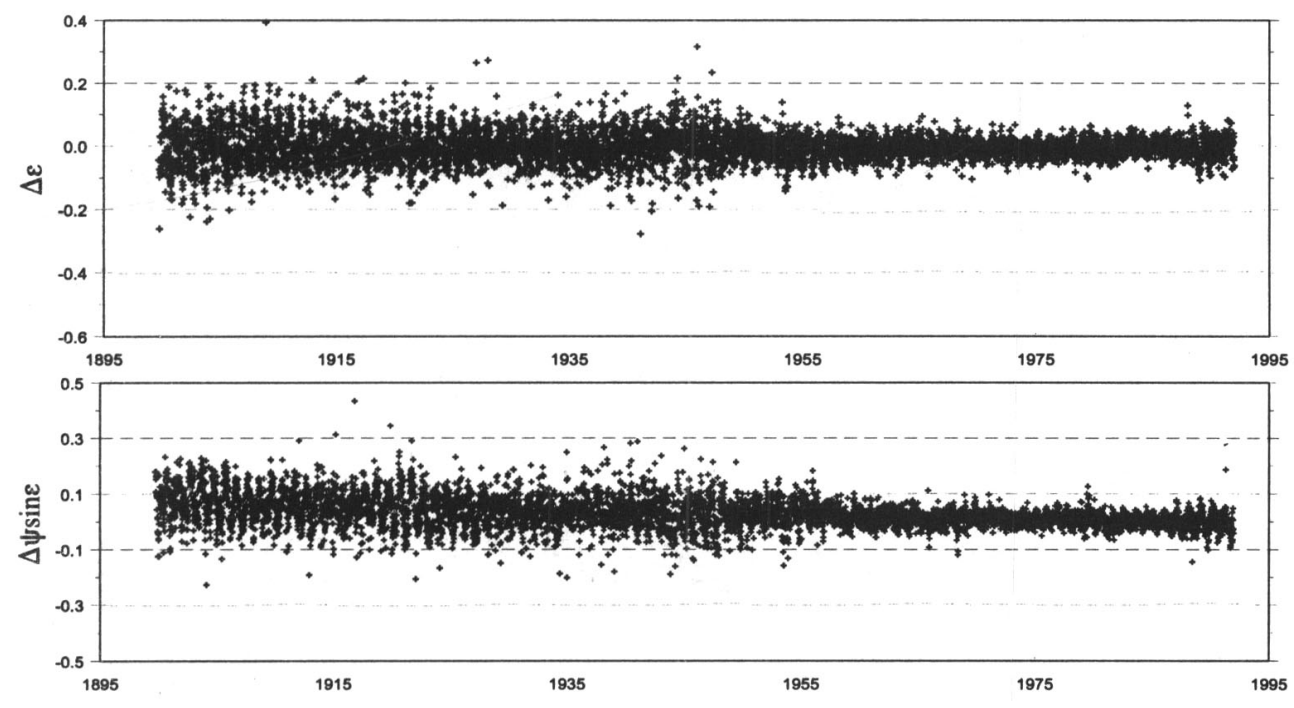

Figure 1. Celestial pole offsets from optical astrometry OA99.

In addition, we derived also a set of auxiliary 'station' parameters, giving the systematic deviations of individual instruments and the rheological parameter $\Lambda=1+k-l$ (responsible for nonrigid tidal variations of local verticals). The list of the instruments used in the solution is given in Table 1.

\section{Celestial pole offsets and their analysis}

Because we used the standard IAU models of precession IAU1976 (Lieske et al., 1977) and nutation IAU1980 (Seidelmann, 1982) to calculate the apparent positions of the observed stars, the celestial pole offsets derived from our solution express the displacements of the Earth's pole from the celestial ephemeris pole whose position is defined by these models. The results are plotted in Figure 1, the values themselves in the upper part (crosses), their formal uncertainties below (lines), both in the same scale. It is necessary to say that $\Delta \varepsilon_{i}, \Delta \psi_{i}$, referred to the same epoch $t_{i}$, are strongly correlated (their error ellipses highly elongated), due to only a partial coverage of observations during a day (for a detailed explanation of this effect see Vondrák et al. (1992). The error ellipses are always pointed towards the Sun (the observations being centred around local midnight) and they thus rotate in the celestial reference frame with an annual period that causes an annual change in rms errors of $\Delta \varepsilon$ and $\Delta \psi$ (phase shifted by $90^{\circ}$ ). This effect became much weaker after 1956, when latitude observations (the only type of observations used before that date) were combined with Universal Time observations. The figure shows also that the noise level substantially diminishes after 1956, thanks to a larger number of participating observatories (and, consequently, also observations).

The series of celestial pole offsets was subject to analysis, in which we used a classical least-squares method to estimate corrections to precession plus the nine most significant nutation terms (OA99/1). Since the resolution of the series 
Table 1. List of participating observatories and instruments in OA99.

\begin{tabular}{l|l|l|rr}
\hline Observatory & Instr. & Interval & long. & lat. \\
\hline Beijing & PAST & $1979-87$ & $116^{\circ} 20^{\prime}$ & $40^{\circ} 06^{\prime}$ \\
Belgrade & ZT & $1949-85$ & 2031 & 4448 \\
Blagoveschtchensk & ZT & $1959-91$ & 12730 & 5019 \\
Bratislava & CZ & $1987-91$ & 1707 & 4809 \\
Carloforte & ZT & $1899-43,46-78$ & 819 & 3908 \\
Cincinnati & ZT & $1899-16$ & 8425 & 3908 \\
Gaithersburg & ZT & $1899-14,32-78$ & 7712 & 3908 \\
Grasse & PAST & $1983-91$ & 656 & 4345 \\
Irkutsk & ZT,PTI & $1958-90,79-91$ & 10420 & 5217 \\
Józefoslaw & ZT & $1961-91$ & 2100 & 5206 \\
Kharkov & PTI & $1973-91$ & 3614 & 5000 \\
Kitab & ZT & $1930-78$ & 6653 & 3908 \\
Mizusawa & ZT,FZT & $1900-78,67-84$ & 14108 & 3908 \\
& PZT\#1,2 & $1959-75,74-91$ & $-"-$ & $-"-$ \\
Mount Stromlo & PZT & $1957-85$ & 14900 & -3519 \\
Nikolaiev & PTI & $1974-91$ & 3159 & 4658 \\
Ondrejov & PZT & $1973-91$ & 1447 & 4955 \\
Paris & AST & $1956-82$ & 220 & 4850 \\
Pecný & CZ & $1970-91$ & 1447 & 4955 \\
Poltava & ZT\#1,2,3 & $1949-90,50-68,68-80$ & 3430 & 4936 \\
Praha & CZ & $1980-84,85-91$ & 1425 & 5005 \\
Pulkovo & ZT & $1904-41,48-91$ & 3020 & 5946 \\
& PTI\#1,2,3 & $1959-71,71-85,71-91$ & $-"-$ & $-"-$ \\
Punta Indio & PZT & $1971-84$ & 5717 & 3521 \\
Richmond & PZT\#1,2 & $1949-87,81-89$ & 8023 & 2536 \\
Santiago & AST & $1965-90$ & 7033 & 3324 \\
Shaanxi & PAST\#1,2 & $1974-84,85-91$ & 10933 & 3457 \\
Shanghai & AST,PAST & $1962-84,75-84$ & 12126 & 3111 \\
Simeiz & AST & $1977-90$ & 3400 & 4424 \\
Tschardjui & ZT & $1899-19$ & 6329 & 3908 \\
Tuorla-Turku & VZT & $1963-89$ & 2230 & 6025 \\
Ukiah & ZT & $1899-60$ & 12313 & 3908 \\
Washington & PZT\#1,2,3 & $1915-55,54-84,81-91$ & 7704 & 3855 \\
Wuchang & AST,PTI & $1964-86,81-86$ & 11421 & 3032 \\
Yunnan & PAST & $1980-91$ & 10248 & 2502 \\
\hline \hline
\end{tabular}

ZT - visual zenith-telescope, VZT - visual zenith tube, FZT - floating zenith-telescope, PZT - photographic zenith tube, PTI - photoelectric transit instrument, AST - Danjon astrolabe, PAST - photoelectric astrolabe, CZ - circumzenithal 
is five days, only the long-periodic terms (between 13.6 days and 18.6 years) are considered. In order to estimate how robust the solution is, we did the same analysis with the most precise part of the solution, covering only 1956.0-1992.0 (OA99/2). Both sets are further compared with the values obtained by analyzing a recent VLBI solution of GSFC (covering only a twenty-year interval, 1979.591999.92), using exactly the same algorithm. The results of all three analyses are depicted in Table 2. The correlations between individual values (except for the constant term that is always correlated with the trend because of the choice of the epoch J2000.0) in the table are rather small for optical astrometry (They do not exceed 0.13 in case of $0 A 99 / 1$ and 0.29 in case of OA99/2.), thanks to the length of the series. It is not the case for VLBI where the correlation is as high as 0.95 between the trend and cosine term of the 18-yr period.

Table 2. Corrections to precession and long-periodic nutation terms, derived from optical astrometry observations (OA99/1 from 1899.71992.0, OA99/2 from 1956.0-1992.0) and VLBI. In the first three rows, sine columns give the trends in milliarcseconds per year, cosine columns the mean celestial offsets at epoch J2000.0.

\begin{tabular}{|c|c|c|c|c|c|}
\hline \multirow{2}{*}{$\begin{array}{l}\text { Argument / } \\
\text { Period [days] }\end{array}$} & & \multicolumn{2}{|c|}{ Longitude [mas] } & \multicolumn{2}{|c|}{ Obliquity [mas] } \\
\hline & & $\sin ($ ('in') & $\cos$ ('out') & $\cos \left(\right.$ ('in') $^{\prime}$ & sin ('out') \\
\hline 0 & OA99/1 & $-2.156 \pm 0.046$ & $-31.02 \pm 1.71$ & $-9.17 \pm 0.64$ & $-0.093 \pm 0.018$ \\
\hline \multirow[t]{2}{*}{$\infty$} & OA $99 / 2$ & $-1.516 \pm 0.143$ & $-16.15 \pm 3.52$ & $-7.12 \pm 1.38$ & $0.007 \pm 0.056$ \\
\hline & VLBI & $-2.990 \pm 0.013$ & $-43.00 \pm 0.10$ & $-4.90 \pm 0.05$ & $-0.220 \pm 0.007$ \\
\hline$\Omega$ & OA99/1 & $-4.31 \pm 1.36$ & $-2.15 \pm 1.39$ & $1.83 \pm 0.50$ & $2.76 \pm 0.50$ \\
\hline \multirow[t]{2}{*}{6798} & OA $99 / 2$ & $-3.84 \pm 1.72$ & $-2.26 \pm 1.81$ & $1.71 \pm 0.69$ & $2.36 \pm 0.66$ \\
\hline & VLBI & $-6.89 \pm 0.03$ & $3.39 \pm 0.07$ & $2.89 \pm 0.04$ & $1.44 \pm 0.01$ \\
\hline $2 \Omega$ & OA99/1 & $3.12 \pm 1.32$ & $2.41 \pm 1 . \overline{33}$ & $-1.10 \pm 0.49$ & $1.22 \pm 0.49$ \\
\hline \multirow[t]{2}{*}{3399} & OA99/2 & $2.28 \pm 1.65$ & $2.97 \pm 1.67$ & $-1.34 \pm 0.66$ & $1.51 \pm 0.65$ \\
\hline & VLBI & $1.08 \pm 0.03$ & $-0.15 \pm 0.02$ & $-0.29 \pm 0.01$ & $0.10 \pm 0.01$ \\
\hline $2 F-2 D+2 \Omega$ & OА99/1 & $-14.50 \pm 1.36$ & $-4.03 \pm 1.39$ & $4.68 \pm 0.50$ & $-2.28 \pm 0.50$ \\
\hline \multirow[t]{2}{*}{182.6} & OA $99 / 2$ & $-13.16 \pm 1.68$ & $-4.12 \pm 1.72$ & $4.45 \pm 0.66$ & $-0.82 \pm 0.65$ \\
\hline & VLBI & $1.57 \pm 0.02$ & $-1.36 \pm 0.02$ & $-0.58 \pm 0.01$ & $-0.43 \pm 0.01$ \\
\hline$l^{\prime}$ & OA99/1 & $-14.15 \pm 1.23$ & $6.58 \pm 1.56$ & $10.69 \pm 0.48$ & $5.99 \pm 0.52$ \\
\hline \multirow[t]{2}{*}{365.3} & OA99/2 & $-4.82 \pm 1.54$ & $8.50 \pm 1.90$ & $9.04 \pm 0.63$ & $6.19 \pm 0.67$ \\
\hline & VLBI & $4.99 \pm 0.01$ & $1.24 \pm 0.01$ & $2.10 \pm 0.01$ & $-0.27 \pm 0.01$ \\
\hline \multirow{3}{*}{$\begin{array}{l}l^{\prime}+2 F-2 D+2 \Omega \\
121.7\end{array}$} & OA99/1 & $-3.82 \pm 1.36$ & $-0.46 \pm 1.37$ & $-1.92 \pm 0.49$ & $-0.08 \pm 0.50$ \\
\hline & OA $99 / 2$ & $-2.75 \pm 1.69$ & $-1.93 \pm 1.70$ & $-0.54 \pm 0.65$ & $0.82 \pm 0.65$ \\
\hline & VLBI & $0.01 \pm 0.01$ & $-0.02 \pm 0.01$ & $0.04 \pm 0.01$ & $-0.05 \pm 0.01$ \\
\hline \multirow{3}{*}{$\begin{array}{l}2 F-2 D+\Omega \\
177.8\end{array}$} & OA99/1 & $3.31 \pm 1.36$ & $2.97 \pm 1.37$ & $-3.69 \pm 0.50$ & $2.80 \pm 0.50$ \\
\hline & OA $99 / 2$ & $3.76 \pm 1.70$ & $4.45 \pm 1.70$ & $-3.80 \pm 0.65$ & $2.75 \pm 0.65$ \\
\hline & VLBI & $-0.13 \pm 0.02$ & $0.01 \pm 0.02$ & $0.11 \pm 0.01$ & $0.01 \pm 0.01$ \\
\hline $2 F+2 \Omega$ & OA99/1 & $-0.18 \pm 1.33$ & $1.37 \pm 1.32$ & $\overline{0.58 \pm 0.49}$ & $-072 \pm 0.49$ \\
\hline \multirow[t]{2}{*}{13.66} & OA99/2 & $-0.90 \pm 1.66$ & $1.36 \pm 1.66$ & $0.68 \pm 0.65$ & $-0.99 \pm 0.65$ \\
\hline & VLBI & $-0.35 \pm 0.02$ & $0.29 \pm 0.02$ & $0.14 \pm 0.01$ & $0.17 \pm 0.01$ \\
\hline T & OA99/1 & $-0.09 \pm 1.32$ & $-0.47 \pm 1.32$ & $-0.73 \pm 0.49$ & $1.04 \pm 0.49$ \\
\hline \multirow[t]{2}{*}{27.55} & OA99/2 & $-0.92 \pm 1.65$ & $-0.25 \pm 1.65$ & $-0.69 \pm 0.65$ & $1.20 \pm 0.65$ \\
\hline & VLB & $-0.13 \pm 0.01$ & $-0.06 \pm 0.01$ & $0.06 \pm 0.01$ & $0.06 \pm 0.01$ \\
\hline $2 F+\Omega$ & OA99/1 & $\overline{2.23 \pm 1.33}$ & $-1.91 \pm 1.32$ & $\overline{0.46 \pm 0.49}$ & $-0.14 \pm 0.50$ \\
\hline \multirow[t]{2}{*}{13.63} & OA99/2 & $3.15 \pm 1.67$ & $-1.19 \pm 1.65$ & $0.91 \pm 0.65$ & $-0.23 \pm 0.65$ \\
\hline & VLBI & $-0.19 \pm 0.02$ & $-0.02 \pm 0.02$ & $0.07 \pm 0.01$ & $0.05 \pm 0.01$ \\
\hline
\end{tabular}




\section{Discussion and conclusions}

First of all, one should notice rather big differences among the trends (sine columns, first three rows) in longitude: 2.156(OA99/1), 1.516(OA99/2) and 2.990(VLBI) and in obliquity: -0.093(OA99/1), 0.007(OA99/2) and 0.220(VLBI). These differences are definitely much larger than their formal errors but, on the other hand, the differences between OA99/1 and VLBI are smaller than those of our preceding solution OA97 (Vondrák et al., 1998b) that yielded $1.543 \mathrm{mas} / \mathrm{yr}$ in longitude and $0.131 \mathrm{mas} / \mathrm{yr}$ in obliquity. The correction to precession is now (solution OA99/1) much closer to the VLBI value than before. This change is very probably due to our less tolerant approach to Hipparcos proper motions (20\% of them being corrected in OA99 in contrast to only $10 \%$ in OA97). Also interesting is the obvious change of trend in longitude around 1956, noticed already in the OA97 solution by Bizouard et al. (1997). This effect can probably be also caused by imperfections of the Hipparcos proper motions. (Different stars were observed in the second half of the century than in the first one.)

Secondly, there is a discrepancy in the cosine (out-of-phase) term of the principal nutation in longitude between both optical solutions and VLBI (they differ in sign). This is the very term that is highly correlated with the trend in the VLBI solution. This also holds for the cosine term in longitude with a period of 3398 days (see also Yaya et al., (2000) for a similar discussion). The largest differences between optical astrometry and VLBI are however found in the semiannual and annual terms. In addition, the values found from the present solution OA99 differ substantially from OA97. It is clear that this is the weakest point of optical astrometry. We suspect that this effect is mostly due to proper motions of some of the stars. Different stars at different right ascensions are observed during the year (Only the 'night' stars are observed.), and if their positions differ from reality at a certain part of the sky they can produce an annual wave in celestial pole offsets. All the other terms agree quite well, on the level of their formal standard errors.

Acknowledgments. This study was made possible thanks to the support through the Key Project No. K1003601 financed by the Academy of Sciences of the Czech Republic.

\section{References}

Bizouard, Ch., Capitaine, N., Ron, C. \& Vondrák, J., 1997, in Proc. IAU Coll. 165 Dynamics and astrometry of natural and artificial celestial bodies, I. Wytrzyszczak, J. Lieske, R.A. Feldman (eds.), Kluwer, 481-486.

Feissel, M. \& Mignard, F., 1998, Astron. Astrophys., 331, L33-L36.

Kovalevsky, J., Lindegren, L., Perryman, M.A.C. et al., 1997, Astron. Astrophys., 323, 620-633.

Lieske, J.H., Lederle, T., Fricke, W. \& Morando B., 1977, Astron. Astrophys., $58,1-16$.

Seidelmann, P.K., 1982, Celest. Mech., 27, 79-106.

Vondrák, J., Feissel, M. \& Essaïf, N., 1992, Astron. Astrophys., 262, 329-340. 
Vondrák, J., Pešek, I., Ron, C. \& Čepek, A., 1998a, Pub. Astron. Inst. Acad. Sci. Czech Rep., 87, 1-56.

Vondrák, J., Ron, C., Pešek, I. \& Čepek A., 1998b, in Journées 1997 Systèmes de référence spatio-temporels, J. Vondrák \& N. Capitaine (eds.), Astron. Inst. Prague \& Observatoire de Paris, 118-121.

Vondrák, J., Ron, C. \& Pešek, I., 2000, in Proc. IAU Coll. 178 Polar motion: historical and scientific aspects, S. Dick, D. McCarthy \& B. Luzum (eds.), ASP, 239-250.

Yaya, P., Bizouard, Ch. \& Ron, C. 2000, in Proc. IAU Coll. 178 Polar motion: historical and scientific aspects, S. Dick, D. McCarthy \& B. Luzum (eds.), ASP, 607-612. 